\title{
Robot-assisted robotic prostatectomy in a patient with dilated cardiomyopathy and LVEF of $35 \%$
}

Volume 8 Issue 5 - 2017

\section{Introduction}

Prostate cancer is the most common cancer in the male population and the third most common cause of death. ${ }^{1}$ Depending on the degree and type of tumor, radical prostatectomy with or without lymphadenectomy is a therapeutic alternative of choice. ${ }^{2}$ Robotassisted laparoscopic radical prostatectomy is increasingly being used in the face of open prostatectomy since oncologically it presents the same results and also has less bleeding, a lower incidence of bladder incontinence and sexual dysfunction, less postoperative pain and a shorter hospital stay. ${ }^{3}$ Robotic prostatectomy requires a position in $45^{\circ}$ extreme trendelembur associated with pneumoperitoneum causes intraoperative hemodynamic changes such as increased peripheral vascular resistance, mild-moderate preload reduction, and decreased cardiac output and vital lung capacity by increased pulmonary atelectasis. $^{4}$

Severe left ventricular dysfunction precludes surgical intervention, so alternatives to improve cardiac function should be sought. An exhaustive adjustment of the treatment, the administration of Levosimendan 48 hours before the surgery and a very careful intraoperative management makes possible the accomplishment of a robotic prostatectomy in a patient with an LVEF of $35 \%$.

\section{Clinical case}

A 65-year-old male with a personal history of hypertension, dyslipidemia, chronic atrial fibrillation, left bundle branch block, dilated cardiomyopathy with a $35 \%$ LVEF of unknown origin, moderate mitral insufficiency, moderate effort dyspnea, NYHA functional class 2 . Coronary angiography was performed to rule out an ischemic origin of normal coronary heart failure. Usual treatment: carvedilol $25 \mathrm{mg} / 24$ hours, enalapril $5 \mathrm{mg} / 24$ hours, simtron, furosemide $20 \mathrm{mg}$ if water decompensation. Cited in a pre-anesthetic consultation, we objected to difficulty in the airway, Mallampatti IV, and a severe myocardial dysfunction with which we consulted with cardiology decided to increase the dose of carvedilol to $25 \mathrm{mg} / 12$ hours and to enter the patient 48 hours before surgery in the ICU to administer Levosimendan at a loading dose of 7 micrograms $/ \mathrm{kg}$. Levosimendan increases calcium sensitivity by cardiac troponin myofilaments by increasing myocardial contractility and decreasing both pulmonary and systemic peripheral resistance in addition to having an antiarrhythmic effect. ${ }^{5}$

The preoperative plan was to have 3 weeks with the new carvelidol regimen, to enter into the ICU the patient 48 hours before the surgery to administer levosimendan and on the day of the surgery to perform an echocardiogram to decide the route of surgical approach if robotic or open prostatectomy. Echocardiography on the day of surgery gave us an LVEF of $51 \%$ with mild mitral insufficiency and a heart rate of $65 \mathrm{bpm}$, so we decided to start laparoscopic surgery with a robot. During the intervention we monitor heart rate, $\mathrm{SO}_{2}$, non-invasive blood pressure, invasive arterial pressure in the radial artery, central venous pressure in the right internal jugular vein and with the pressure

\author{
Alba de Caceres E, Gonzalez Elisabeth, Alba \\ de Caceres M, Mira Betancurt Adrian \\ Servicio de Anestesiologia, Hospital Universitario Sanitas La \\ Zarzuela, Spain
}

Correspondence: Alba de Caceres E, Servicio de Anestesiologia, Hospital Universitario Sanitas La Zarzuela, Spain, Email enriquealbac@gmail.com

Received: May 23, 2017 | Published: September 26, 2017

transducer housed in the right midline axillary at the auricle height so that no was altered with the trendelembur angulation. We channel two peripheral tracks of the $16 \mathrm{G}$ caliber in addition to the central track of 2 lights. We monitor muscle relaxation with a TOF monitor with the possibility of measuring tetany and hypnosis with bispectral index (BIS).

The first measurement of PVC was -1, giving 1 liter of crystalloids and $500 \mathrm{ml}$ of hydroxyethyl starch $6 \% 130 / 0.4$, just prior to induction of anesthesia until the PVC was positive. The patient was premedicated with $2 \mathrm{mg}$ midazolam and $0.10 \mathrm{mg}$ fentanyl, preoxigenated with face mask for 5 minutes and anesthetic induction was performed with $15 \mathrm{mg}$ etomidate. $50 \mathrm{mg}$ of rocuronium bromide was used as a muscle relaxant and the patient was intubated with a 8 -gauge orotracheal tube with a guarantor with a Cormack 3 . During anesthetic induction, L-noradrenaline at 0.05 micrograms $/ \mathrm{kg} / \mathrm{min}$ and dobutamine at 5 micrograms $/ \mathrm{kg} / \mathrm{min}$ were administered via the central lane to avoid severe hypotension during induction. Once stabilized the patient proceeded to place a mesh between the intestinal block and the bladder to be able to perform the intervention with a minimum trendelembur of $20^{\circ}$. When the pneumoperitoneum was inflated, the patient had an extreme bradycardia of $32 \mathrm{lpm}$, which forced us to cease pneumoperitoneum and administer $1 \mathrm{mg}$ of atropine, causing a tachycardia of $110 \mathrm{bpm}$ with hemodynamic stability. Once the heart rate was stabilized, the pneumoperitoneum was slowly reinflated to a pressure of $10 \mathrm{~mm} \mathrm{Hg}$, this time keeping the heart rate and blood pressure stable, finally being inclined to the patient $15^{\circ}-20^{\circ}$ of trendelemburg and the surgery.

Muscle relaxation was monitored every 5 min with TOF and the objective was to maintain a TOF of less than 2 to have an acceptable laparoscopic bell with inflating pressures of 9-10mm $\mathrm{Hg}$, for which we administered Rocuronium Bromide on demand. Analgesia was achieved with fentanyl on demand according to hemodynamic values, and maintenance was performed with $2 \%$ sevofluorane adjusted to achieve BIS monitoring between 45 and 55. The surgery was more diicultosa than normal and the duration was 4 hours. During surgery 
there was no need to transfuse hematite concentrates, maintenance fluid therapy was restrictive at a rate of $250 \mathrm{ml} / \mathrm{h}$ of crystalloids and hemodynamic variability was managed by adjusting the doses of L-Noradrenaline and dobutamine. The patient was transferred intubated and sedoanalgesiado to the ICU, hemodynamically stable with vasoactive drugs at testimonial doses. He was awakened and extubated at 12 hours in the ICU without incident and was discharged from the ICU within 48 hours of admission. In plant, did not have to transfuse, developed heart failure by water decompensation that was treated with diuretics (furosemide) without further incidences. He maintained good cardiovascular function, was admitted for 7 days and was discharged without incident.

\section{Discussion}

Although the treatment of choice in prostate cancer is radical prostatectomy and the technique with the best oncological and functional results is robot-assisted prostatectomy, the cardiac function and the general state of the patient was limiting factors of operability. ${ }^{6}$ Severe left ventricular dysfunction with an LVEF of $35 \%$ prevents adequate blood pumping due to the increased peripheral resistance generated by the pneumoperitoneum, and the acute decrease in preload caused by compression of the inferior vena cava causes the heart to Collapse causing extreme bradycardia with cardiac arrest. We had to optimize the cardiovascular function of the patient, to avoid the position of extreme trendelembur and to use the lowest pneumoperitoneum insufflation pressure possible. The optimization of the cardiac function is achieved with levosimendan, a drug that increases the response of myofilaments to calcium by binding to cardiac troponin $\mathrm{C}$ and also acts on the potassium channels sensitive to ATP in the vascular smooth muscle fiber causing peripheral and coronary vasodilatation. Therefore, the effects of this drug are on the one hand an increase in contractility and on the other, a decrease in systemic and pulmonary vascular resistance. Levosimendan enhances contractility without increasing the demand for oxygen but causes a very important generalized vasoplegia that we had to correct it with a mixed preload of crystalloids and colloids before inflating the pneumoperitoneum. The positive inotropic effect of Levosimendan in single dose persists for at least one week and must be administered at least 48 hours before surgery because its effect begins after the second day of administration. ${ }^{7}$

To maintain a pneumoperitoneum as light as possible we obtained an extreme muscular relaxation with rocuronium bromide on demand, administering muscle relaxant as the TOF marked us 2 of four in this way thanks to the trendelembur position of $20^{\circ}$ and extreme relaxation we managed to perform the Intervention with pressures in the pneumoperitoneum of $9-10 \mathrm{mmHg} .{ }^{8}$ The very low pneumoperitoneum pressure helps to improve the filling of the right atrium, it helps the contractility of the left ventricle and improve the cardiac function and also decressing peripheral resistances. A 9-10 $\mathrm{mm} \mathrm{Hg}$ pression of Pneumoperitoneum considerably reduces the amount of $\mathrm{CO}_{2}$ that travel from peritoneum to blood decreasing the respiratory acidosis which could appear in some laparoscopy surgery.

\section{Conclusion}

Levosimendan improved cardiac function from $35 \%$ to $50 \%$. Leosimendan must be administered at least 3 days before the surgery date because cause a very important vasoplegia. Robotic Prostatectomy need an important preload with colloids and cristaloids fluids to preserve cardiac function while pneumoperitoneum is doing. Extreme muscle relaxation is recommended to use less pression and less $\mathrm{CO}_{2}$ gas flow during pneumoperitoneum so patient had less postoperative pain and better haemodinamic function. Robotic prostatectomy can be the gold standard treatment in prosata cancer despite patient have a very depressed cardiac function.

\section{Acknowledgments}

None.

\section{Conflicts of interest}

Authors declare that there is no conflict of interest.

\section{References}

1. Galceran J, Ameijide A, Carulla M, et al. Cancer incidence in Spain, 2015. Clin Transl Oncol. 2017;19(7):799-825.

2. Heidenreich A, Bellmunt J, Bolla M, et al. EAU guidelines on prostate cancer. Part 1: screening, diagnosis, and treatment of clinically localised disease. Eur Urol. 2011;59(1):61-71

3. Jacobsen NE, Moore KN, Estey E, et al. Open versus laparoscopic radical prostatectomy: a prospective comparison of postoperative urinary incontinence rates. J Urol. 2007;177(2):615-619.

4. Rosendal C, Markin S, Hien M, et al. Cardiac and hemodynamic consequences during capnoperitoneum and steep Trendelenburg positioning: lessons learned from robot-assisted laparoscopic prostatectomy. J Clin Anesth. 2014;26(5):383-389.

5. Bozhinovska M, Taleska G, Fabian A, et al. The Role of Levosimendan in Patients with Decreased Left Ventricular Function Undergoing Cardiac Surgery. Open Access Maced J Med Sci. 2016;4(3):510-516.

6. Jesús Moreno Sierra, Carlos Núñez Mora, Ma Isabel Galante Romo, et al. Prostatectomía radical asistida por robot Da Vinci®: Un año de experiencia en el Hospital Clínico San Carlos. Arch Esp Urol. 2008;61(3):385-396.

7. Landoni G, Lomivorotov VV, Alvaro G, et al. Levosimendan for Hemodynamic Support after Cardiac Surgery. $N$ Engl J Med. 2017;376:2021-2031.

8. Barrio J, Errando CL, San Miguel G, et al. Effect of depth of neuromuscular blockade on the abdominal space during pneumoperitoneum establishment in laparoscopic surgery. J Clin Anesth. 2016;34:197-203. 